\title{
Dietary fish oil preserves cardiac function in the hypertrophied rat heart
}

\author{
Peter L. McLennan ${ }^{1 *}$, Mahinda Y. Abeywardena ${ }^{2}$, Julie A. Dallimore ${ }^{2}$ and Daniel Raederstorff ${ }^{3}$ \\ ${ }^{1}$ Cardiovascular Research Laboratory, Graduate School of Medicine, University of Wollongong, Wollongong, \\ NSW 2522, Australia \\ ${ }^{2}$ CSIRO Human Nutrition, Adelaide, Australia \\ ${ }^{3}$ DSM Nutritional Products, Basel, Switzerland \\ (Submitted 13 June 2011 - Final revision received 18 September 2011 - Accepted 30 September 2011 - First published online 9 November 2011)
}

\section{Abstract}

Regular fish or fish oil intake is associated with a low incidence of heart failure clinically, and fish oil-induced reduction in cardiac remodelling seen in hypertrophy models may contribute. We investigated whether improved cardiac energy efficiency in non-hypertrophied hearts translates into attenuation of cardiac dysfunction in hypertrophied hearts. Male Wistar rats ( $n$ 33) at 8 weeks of age were shamoperated or subjected to abdominal aortic stenosis to produce pressure-overload cardiac hypertrophy. Starting 3 weeks post-operatively to follow initiation of hypertrophy, rats were fed a diet containing $10 \%$ olive oil (control) or 5\% fish oil (ROPUFA 30 (17\% EPA, $10 \%$ DHA) $)+5 \%$ olive oil (FO diet). At 15 weeks post-operatively, ventricular haemodynamics and oxygen consumption were evaluated in the blood-perfused, isolated working heart. Resting and maximally stimulated cardiac output and external work were $>60 \%$ depressed in hypertrophied control hearts but this was prevented by FO feeding, without attenuating hypertrophy. Cardiac energy efficiency was lower in hypertrophy, but greater in FO hearts for any given cardiac mass. Coronary blood flow, restricted in hypertrophied control hearts, increased with increasing work in hypertrophied FO hearts, revealing a significant coronary vasodilator reserve. Pronounced cardiac dysfunction in hypertrophied hearts across low and high workloads, indicative of heart failure, was attenuated by FO feeding in association with membrane incorporation of $n$-3 PUFA, principally DHA. Dietary fish oil may offer a new approach to balancing the high oxygen demand and haemodynamic requirements of the failing hypertrophied heart independently of attenuating hypertrophy.

\section{Key words: Fish oil: $n$-3 Fatty acids: Heart failure: Hypertrophy: Diet}

Despite declining heart disease mortality rates, a continuing rise in heart failure incidence is of major clinical concern in many nations ${ }^{(1)}$. Regular fish (and hence $n$-3 PUFA) consumption is associated with low CVD mortality ${ }^{(2,3)}$. Antiarrhythmic effects first identified in animal models ${ }^{(4)}$ are evident in clinical studies showing reduced incidence of primary cardiac arrest ${ }^{(5)}$ and post-infarction arrhythmic deaths ${ }^{(6)}$, and represent the best-supported mechanism of action. However, in addition to arrhythmia prevention, alternative mechanisms of fish oil action, such as prevention of heart failure, are implicated directly from epidemiology ${ }^{(7)}$, and indirectly as reduced non-arrhythmic but otherwise unspecified cardiac deaths and post-infarction deaths ${ }^{(8)}$. These clinical outcomes are independent of dietary $n$ - 3 PUFA effects on classic risk factors such as plasma TAG and thrombogenic factors ${ }^{(9)}$ or blood pressure $^{(8,10)}$. Animal studies have described fish oil-induced improvements in heart function and the prevention of fatal arrhythmia in non-disease states that are associated with the incorporation of the long-chain n-3 PUFA DHA into myocardial membranes ${ }^{(4,11-14)}$ and occur at lower intakes than for the vascular effects ${ }^{(15)}$.

Hearts isolated from rats fed fish oil exhibit significantly reduced oxygen consumption without compromise to cardiac output (CO) or external work ${ }^{(13)}$. This represents increased energy efficiency of oxygen use, increased coronary perfusion reserve, less damage in acute ischaemia and reduced myocardial stunning ${ }^{(13,16,17)}$. Cardiac hypertrophy is generally characterised by morphological and biochemical changes that predispose to myocardial ischaemia through disturbances of both oxygen supply and demand at the cellular level, which promote energetic failure and heart failure ${ }^{(18)}$. For example, high ventricular wall tension increases oxygen demand and restricts coronary blood flow, compromising oxygen delivery to myocardial mitochondria already jeopardised by high inter-capillary distances. The capacity of the hypertrophied cell to use oxygen has reportedly been further reduced by

Abbreviations: CO, cardiac output; FO, fish oil; FOH, rats with cardiac hypertrophy fed with a fish oil diet; $\mathrm{MVO}_{2}$, myocardial oxygen consumption; OO, olive oil; $\mathrm{OOH}$, rats with cardiac hypertrophy fed with an olive oil diet; SV, stroke volume. 
a high ratio of contractile protein:mitochondria, and mitochondrial function itself may be compromised ${ }^{(18)}$. Consequently, an intervention that reduces myocardial oxygen consumption $\left(\mathrm{MVO}_{2}\right)$ without detriment to myocardial work output could improve cardiac function in hypertrophy and retard the progression to failure ${ }^{(19)}$.

The present study tests the hypothesis that incorporation of $n$-3 PUFA into myocardial membranes through fish oil feeding will improve cardiac energy efficiency and attenuate cardiac dysfunction and heart failure in the hypertrophied heart.

\section{Methods}

A total of thirty-three male Wistar rats (CSIRO Human Nutrition, Adelaide, Australia) were used in the present study. The experiments were conducted according to the National Health and Medical Research Council of Australia, Guidelines for the Use of Experimental Animals, and were approved by the Animal Care and Ethics Committees of the University of Wollongong and CSIRO Human Nutrition.

\section{Animal model of pressure-overload hypertrophy}

Under ketamine $(70 \mathrm{mg} / \mathrm{kg})+$ xylazine $(5 \mathrm{mg} / \mathrm{kg})$ anaesthesia, twenty-eight rats were subjected to restrictive banding of the suprarenal abdominal aorta at 8 weeks of age using a blunt $23 \mathrm{G}$ needle as a template. The needle was tied tightly against the aorta above the renal arteries (causing visible kidney blanching), and then removed, leaving the suture in place to partially restore blood flow (visually confirmed). Another five rats were subjected to sham operation without aortic restriction. Heart function was assessed 15 weeks post-operatively.

Cardiac hypertrophy was determined from the heart weight: body-weight ratio and the heart weight:tibia length ratio. The latter index is not distorted by acute fluctuations in body weight or body-weight gain from fluid retention. Cardiac hypertrophy was defined as a ratio $>2$ SD above the mean for rats of the same strain and age as those in the present study. The pooled mean of ventricle dry weight:tibia length from fifty-three rats previously recorded in this laboratory, not subjected to surgical intervention, was 0.376 (SD $0.045) \mathrm{g} / \mathrm{cm}$. Cardiac hypertrophy was therefore defined for this strain and age of rat as a ventricle weight:tibia length ratio $>0.466 \mathrm{~g} / \mathrm{cm}$.

The pressure overload induced by aortic stenosis provokes a hypertrophic response within $4 \mathrm{~d}$, and it has been well established by 3 weeks ${ }^{(20)}$. Because fish oil inhibits cardiac hypertrophy when presented before and during a hypertrophic stimulus $^{(21,22)}$, in the present study, the initiation of fish oil feeding was delayed until 3 weeks after surgery in order to focus interpretation on pathophysiology of the hypertrophied heart. The aortic stenosis model was preferred to alternative models of the following: aortic valve disruption (volume overload), which is unsuitable for evaluation in the isolated working heart preparation; and coronary artery occlusion (chronic myocardial infarction), which is prone to a high incidence of acute fatal arrhythmia and potentially confounding effects of tissue repair processes that may be influenced by dietary intervention independently of the effects on hypertrophic function.

\section{Diets}

Rats were fed an olive oil (OO) control diet for 4 weeks before surgery and 3 weeks post-operatively. They were then randomly allocated to either the fish oil (FO) diet or the OO control diet for 12 weeks. Sham-operated rats were fed the OO control diet throughout the experiment. Isoenergetic fully fabricated diets were prepared ${ }^{(16,23)}$ containing $100 \mathrm{~g} / \mathrm{kg}$ diet as fat. The OO (control) diet contained 10\% (by weight) olive oil, and the FO diet contained $5 \%$ olive oil plus 5\% fish oil. The olive oil was commercially available food-grade olive oil, refined to exclude antioxidant phytochemicals. The fish oil (ROPUFA ${ }^{\circledR} 30 n$-3 EPA oil; DSM Nutritional Products, Basel, Switzerland) contained $16 \cdot 8 \%$ EPA (20:5n-3) and $9 \cdot 7 \%$ DHA (22: $6 n-3 ; 32 \cdot 5 \%$ total $n$-3 fatty acids). Olive oil was selected for the isoenergetic control diet on the basis of the following: its main component (monounsaturated oleic acid (18:1)) being a physiologically neutral fat; and the oil providing sufficient PUFA to avoid essential fatty acid deficiency $^{(16)}$. Although $n-3$ PUFA replace $n-6$ PUFA in myocardial membranes, the long-chain $n$-3 PUFA are incorporated in direct relationship to their absolute quantities in the diet and independently of dietary MUFA or n-6 PUFA dietary concentrations ${ }^{(23)}$. The main fatty acid components of the two diets were as follows: total SFA (OO 14\%; FO 22\%); total PUFA (OO 10\%; FO 22\%); oleic acid (18:1; OO $76 \%$; FO $45 \%)$; linoleic acid (18:2n-6; OO $8.3 \%$; FO $5 \cdot 2 \%)$; EPA (FO 8.4\%); DHA (FO 4.9\%).

\section{Isolated working heart}

Rats were anaesthetised (pentobarbitone sodium $60 \mathrm{mg} / \mathrm{kg}$ intraperitoneally), and the right common carotid artery and the left femoral artery were cannulated to record arterial blood pressures proximal and distal to the aortic stenosis. Rats were then killed by rapid exsanguination and the hearts removed into ice-cold saline and prepared for working heart perfusion with porcine erythrocyte buffer ( $40 \%$ haematocrit in Krebs Henseleit solution) as described previously ${ }^{(13)}$. Briefly, the aorta, left atrium and pulmonary artery were cannulated and the heart perfused with Krebs Henseleit solution in Langendorff mode for $10 \mathrm{~min}$ for rat blood washout. Hearts were switched to working heart mode and perfused with erythrocyte buffer via the left atrium with $10 \mathrm{mmHg}$ filling pressure (preload) and $75 \mathrm{mmHg}$ diastolic aortic pressure (afterload, coronary perfusion pressure). A catheter introduced through silicone self-sealing tubing supplying the left atrium and fed into the ventricle via the mitral valve recorded left ventricular pressure. Working hearts were allowed to stabilise for $15 \mathrm{~min}$.

\section{Cardiac function}

CO and coronary flow were measured at 5 min intervals by timed overflow, with coronary and aortic samples collected 
for the measurement of arterial and venous blood gases and $\mathrm{pH}$. Stroke volume (SV) was calculated from the $\mathrm{CO}$ and heart rate. $\mathrm{MVO}_{2}$ was derived from arterio-venous oxygen differences per unit coronary flow, and external work was derived from the $\mathrm{CO}$ and developed pressure $(\mathrm{CO} \times$ mean aortic pressure) as described previously ${ }^{(13)}$. Cardiac energy efficiency (\%) was calculated as the ratio of energy derived from $\mathrm{MVO}_{2}$ (in $\mathrm{kJ}$ ) to energy delivered as external work (in $\mathrm{kJ}) \times 100$. After equilibration, preload was changed over the range $5-20 \mathrm{mmHg}$ by altering ventricular filling pressure, before returning pressure to $10 \mathrm{mmHg}$ for recovery. Intraventricular pressure, aortic pressure and electrocardiogram were recorded continuously, while coronary and aortic flows were measured and blood samples were collected at 5 min intervals.

\section{Membrane fatty acids}

Hearts were rinsed in ice-cold saline, blotted dry and weighed. A small section of the ventricle free wall was weighed and retained for dry-weight estimation. The remaining ventricle was rapidly frozen using liquid $\mathrm{N}_{2}$-cooled clamps and then frozen at $-60^{\circ} \mathrm{C}$ until required for fatty acid measurement according to established methods ${ }^{(23)}$. In brief, samples of the frozen tissue were pulverised over liquid $\mathrm{N}_{2}$ and washed with centrifugation to remove sucrose. Lipids were extracted using chloroform-methanol with aliquots applied to silicic acid columns to separate neutral lipids and phospholipids. Fatty acid methyl esters of the phospholipids were prepared and extracted for analysis by GC. Fatty acids were identified from their retention times compared with those of authentic methyl fatty acid standards.

\section{Statistical analysis}

The CO, external work, coronary flow, oxygen extraction and $\mathrm{MVO}_{2}$ were expressed $1 / \mathrm{g}$ ventricle dry weight. Results are expressed as means with their standard errors. For haemodynamic measures, two-way ANOVA was conducted with diet and hypertrophy main effects and diet $\times$ hypertrophy interaction. Individual comparisons between diet $\times$ hypertrophy were conducted using Scheffe's post hoc $F$ test for multiple comparisons of individual means. Values in tables sharing a common superscript letter were not significantly different. The level of significance was considered at $P<0 \cdot 05$.

\section{Results \\ Heart and body weights, blood pressure and cardiac
hypertrophy}

Cardiac enlargement sufficient to classify as hypertrophy occurred in six of the fourteen rats in each of the dietary groups subjected to abdominal aortic stenosis. Rats with cardiac hypertrophy (designated $\mathrm{OOH}$ if fed the olive oil diet and $\mathrm{FOH}$ if fed the FO diet) had significantly greater ventricle weights and body weights than non-hypertrophied (designated $\mathrm{OO}$ or FO) or sham-operated rats (Table 1). Tibia length, body weight, body size or heart weight were not different between the dietary groups within either the hypertrophy or non-hypertrophy animals (Table 1). Less variation in tibia length than in body weight resulted in the ventricle weight:tibia length being the clearer discriminator of hypertrophy.

Table 1. Effect of diet and cardiac hypertrophy on animal characteristics and isolated working heart function at equilibrium

(Mean values with their standard errors)

\begin{tabular}{|c|c|c|c|c|c|c|c|c|c|c|}
\hline \multirow[t]{3}{*}{ Diet group... } & \multicolumn{4}{|c|}{$\mathrm{OO}$} & \multirow{2}{*}{\multicolumn{2}{|c|}{$\begin{array}{c}\mathrm{OOH} \\
\begin{array}{c}\text { Hypertrophy } \\
(n 6)\end{array}\end{array}$}} & \multirow{2}{*}{\multicolumn{2}{|c|}{$\frac{\mathrm{FO}}{\begin{array}{c}\text { No hypertrophy } \\
(n 8)\end{array}}$}} & \multirow{2}{*}{\multicolumn{2}{|c|}{$\begin{array}{c}\text { FOH } \\
\begin{array}{l}\text { Hypertrophy } \\
(n 6)\end{array}\end{array}$}} \\
\hline & \multicolumn{2}{|c|}{$\begin{array}{l}\text { Sham control } \\
\quad(n 5)\end{array}$} & \multicolumn{2}{|c|}{$\begin{array}{l}\text { No hypertrophy } \\
(n 8)\end{array}$} & & & & & & \\
\hline & Mean & SEM & Mean & SEM & Mean & SEM & Mean & SEM & Mean & SEM \\
\hline Body weight (g) & 477 & 10 & 442 & 13 & 504 & 28 & 468 & 15 & 486 & 16 \\
\hline Atria weight (g) & 0.12 & 0.01 & 0.12 & 0.01 & 0.19 & 0.03 & 0.12 & 0.01 & 0.15 & 0.02 \\
\hline Ventricle weight $(\mathrm{g})$ & $1.66^{\mathrm{b}}$ & 0.06 & $1.49^{b}$ & 0.05 & $2 \cdot 23^{a}$ & $0 \cdot 10$ & $1.69^{b}$ & 0.06 & $2 \cdot 10^{\mathrm{a}}$ & 0.07 \\
\hline Ventricle dry weight (g) & $0.27^{\mathrm{b}}$ & 0.01 & $0.27^{\mathrm{b}}$ & 0.01 & $0.39^{\mathrm{a}}$ & 0.01 & $0.31^{\mathrm{b}}$ & 0.01 & $0.38^{\mathrm{a}}$ & 0.02 \\
\hline Dry:wet left ventricle weight & 0.163 & 0.005 & 0.185 & 0.004 & 0.175 & 0.007 & 0.188 & 0.002 & 0.184 & 0.002 \\
\hline Tibia length $(\mathrm{cm})$ & $4 \cdot 16$ & 0.01 & $4 \cdot 15$ & 0.04 & $4 \cdot 13$ & 0.07 & $4 \cdot 26$ & 0.07 & $4 \cdot 16$ & 0.06 \\
\hline Ventricle weight:tibia length (g/cm) & $0.400^{\mathrm{b}}$ & 0.021 & $0.359^{b}$ & 0.012 & $0.545^{a}$ & 0.030 & $0.397^{\mathrm{b}}$ & 0.015 & $0.503^{\mathrm{a}}$ & 0.014 \\
\hline Ventricle weight:body weight $(\mathrm{g} / 100 \mathrm{~g})$ & $0.348^{\mathrm{b}}$ & 0.010 & $0.338^{\mathrm{b}}$ & 0.011 & $0.455^{\mathrm{a}}$ & 0.031 & $0.363^{b}$ & 0.017 & $0.436^{\mathrm{a}}$ & 0.032 \\
\hline Heart weight:body weight $(\mathrm{g} / 100 \mathrm{~g})$ & $0.373^{\mathrm{b}}$ & 0.014 & $0.356^{\mathrm{b}}$ & 0.014 & $0.488^{\mathrm{a}}$ & 0.039 & $0.386^{\mathrm{b}}$ & 0.022 & $0.443^{\mathrm{a}}$ & 0.039 \\
\hline Carotid systolic BP $(\mathrm{mmHg})$ & $146^{\mathrm{b}}$ & 14 & $161^{\mathrm{b}}$ & 19 & $220^{\mathrm{a}}$ & 20 & $166^{\mathrm{b}}$ & 17 & $218^{\mathrm{a}}$ & 19 \\
\hline Carotid diastolic BP (mmHg) & $118^{\mathrm{b}}$ & 13 & $131^{\mathrm{b}}$ & 17 & $165^{\mathrm{a}}$ & 13 & $131^{\mathrm{b}}$ & 12 & $164^{\mathrm{a}}$ & 15 \\
\hline Femoral systolic BP (mmHg) & $166^{\mathrm{a}}$ & 13 & $134^{\mathrm{b}}$ & 16 & $136^{\mathrm{b}}$ & 13 & $135^{\mathrm{b}}$ & 19 & $148^{\mathrm{a}, \mathrm{b}}$ & 15 \\
\hline $\begin{array}{l}\text { Femoral diastolic BP (mmHg) } \\
\text { Isolated working heart at equilibrium }\end{array}$ & 125 & 16 & 100 & 14 & 111 & 12 & 114 & 16 & 118 & 14 \\
\hline Heart rate (beats per min) & 207 & 26 & 203 & 23 & 185 & 24 & 211 & 14 & 210 & 19 \\
\hline Cardiac output (ml/min per g dry weight) & $82^{b, c}$ & 19 & $108^{\mathrm{a}, \mathrm{b}}$ & 22 & $56^{\mathrm{c}}$ & 20 & $128^{a, b}$ & 33 & $127^{\mathrm{a}}$ & 20 \\
\hline Coronary flow (ml/min per g dry weight) & $33 \cdot 7^{\mathrm{a}}$ & 4.7 & $34 \cdot 3^{\mathrm{a}}$ & $6 \cdot 2$ & $17 \cdot 3^{b}$ & 3.9 & $30 \cdot 2^{\mathrm{a}}$ & $6 \cdot 1$ & $40 \cdot 6^{a}$ & $4 \cdot 8$ \\
\hline Ventricle $\mathrm{d} P / \mathrm{d} t_{\max }(\mathrm{mmHg} / \mathrm{s})$ & 3070 & 194 & 3634 & 503 & 2389 & 452 & 3233 & 388 & 3236 & 317 \\
\hline Ventricle $-\mathrm{d} P / \mathrm{d} t_{\max }(\mathrm{mmHg} / \mathrm{s})$ & $2460^{b}$ & 269 & $2608^{b}$ & 355 & $1632^{\mathrm{a}}$ & 278 & $2351^{b}$ & 292 & $2560^{b}$ & 320 \\
\hline
\end{tabular}

$\mathrm{OO}$, olive oil diet; $\mathrm{OOH}$, rats with cardiac hypertrophy fed with an olive oil diet; FO, fish oil diet; FOH, rats with cardiac hypertrophy fed with a fish oil; BP, blood pressure. ${ }_{\mathrm{a}, \mathrm{b}, \mathrm{c}}$ Mean values within a row with unlike superscript letters were significantly different $(P<0.05)$. 
Femoral artery pressure was significantly lower $(P<0 \cdot 05)$ in rats subjected to aortic stenosis than in sham-operated controls and did not differ between the rats with or without cardiac hypertrophy or between the dietary groups (Table 1). Carotid artery pressure was significantly higher in animals with cardiac hypertrophy compared with those that failed to develop hypertrophy or sham-operated controls $(P<0.05)$. There were no significant differences in carotid blood pressures between the dietary groups.

\section{Equilibrium heart function}

Under initial conditions of the $10 \mathrm{mmHg}$ preload and the $75 \mathrm{mmHg}$ afterload, there were no differences in spontaneous resting heart rate (Table 1$)$. Uncorrected $\mathrm{CO}(\mathrm{ml} / \mathrm{min})$ was significantly higher in hearts from fish oil-fed rats (FO 41.3 (SEM 10.9); FOH 48.1 (SEM 10.5)) than in hearts from rats fed olive oil (sham 20.1 (sem 4.7); OO 29.0 (sem 3.9); OOH 21.9 (SEM 7.6) $(P<0.01)$, with no significant interaction $(P>0.05)$. When corrected for ventricular mass, the CO of the hypertrophied $\mathrm{OOH}$ hearts was significantly lower per gram of tissue $(P<0.05)$ than the OO, FO and hypertrophied FOH hearts (Table 1). The $\mathrm{CO}$ of the $\mathrm{FOH}$ (hypertrophied) hearts was not significantly different from that of the FO (non-hypertrophied) hearts. There was a significant (diet $\times$ hypertrophy) interaction for the maximum rate of ventricular relaxation $(P=0.02)$ and the $\mathrm{OOH}$ hearts showed a significantly slower relaxation rate than all the other hearts $(P<0 \cdot 05$; Table 1$)$.

Coronary flow did not differ among the non-hypertrophied hearts but was significantly lower in the $\mathrm{OOH}$ hearts $(P<0 \cdot 01)$ than all the other hearts (Table 1 ). The $\mathrm{MVO}_{2}$ at equilibrium in the non-hypertrophied hearts did not differ between the dietary groups or the sham-operated control but was higher in the $\mathrm{FOH}$ hearts and lower in the $\mathrm{OOH}$ hearts relative to all the other hearts $(P<0.05$; Fig. 1$)$. External work was significantly lower in the $\mathrm{OOH}$ hearts $(P<0.01)$ than in the OO, FO and FOH hearts (Fig. 1).

The hypertrophied hearts had significantly lower energy efficiency than the non-hypertrophied hearts (two-way ANOVA (diet, hypertrophy), $P<0.05$ ) and the FO hearts had higher cardiac efficiency than the OO hearts $(P<0.05)$.
Cardiac energy efficiency was significantly lower in the $\mathrm{OOH}$ hearts than in the OO, FO and FOH hearts (Fig. 1).

\section{Cardiac function under load}

The CO, SV (Fig. 2) and external work (Fig. 3) increased with increasing filling pressure (preload) $(P<0 \cdot 0001)$. The hypertrophied hearts had a lower SV, CO or external work over the range of filling pressures $(P<0 \cdot 0001)$. A significant (diet $X$ hypertrophy) interaction was evident for SV $(P=0 \cdot 01)$, CO $(P=0.023)$ and external work $(P=0 \cdot 044)$. The $\mathrm{OOH}$ hearts had a significantly lower SV $(P<0 \cdot 0001), \mathrm{CO}(P<0 \cdot 0001)$ and external work $(P<0.0001)$ than the OO hearts, while there were no significant differences between the $\mathrm{FOH}$ and $\mathrm{FO}$ hearts (SV $P=0 \cdot 10$; CO $P=0.082$; external work $P=0.51$ ). Among the hypertrophied hearts, the $\mathrm{FOH}$ group had a significantly higher SV $(P=0.008), \mathrm{CO}(P=0.003)$ and external work $(P=0.003)$ than the $\mathrm{OOH}$ hearts across the range of filling pressures.

Coronary flow and $\mathrm{MVO}_{2}$ increased with increasing filling pressure ( $P<0.04$; Fig. 2). A significant (diet $\times$ hypertrophy) interaction was evident for coronary flow $(P=0 \cdot 01)$ and $\mathrm{MVO}_{2}$ $(P=0.002)$. Coronary flow over the range of filling pressures was significantly lower in the $\mathrm{OOH}$ hearts than in the $\mathrm{FOH}$ $(P=0.015)$ and $\mathrm{OO}$ hearts $(P=0.0005)$. The $\mathrm{MVO}_{2}$ was not significantly different in the $\mathrm{FOH}$ hearts compared with the FO hearts $(P=0 \cdot 19)$, and there were no significant differences in coronary flow $(P=0 \cdot 12)$ or $\mathrm{MVO}_{2}(P=0.059)$ between the OO and FO hearts. The cardiac energy efficiency of oxygen conversion into external work increased $(P=0 \cdot 0003)$ as external work was increased by increasing filling pressure (Fig. 3), and it was significantly lower in hypertrophy $(P<0 \cdot 0001)$ with both diets. No significant (diet $\times$ hypertrophy) interaction was evident for cardiac energy efficiency $(P=0 \cdot 87)$. Cardiac efficiency was higher in the FO hearts than in the OO and sham hearts $(P=0.004)$ and higher in the $\mathrm{FOH}$ hearts than in the $\mathrm{OOH}$ hearts $(P=0 \cdot 004)$.

\section{Membrane composition}

The phospholipid fatty acid composition of the rat hearts was significantly influenced by the fat source in the diet. The FO (a)

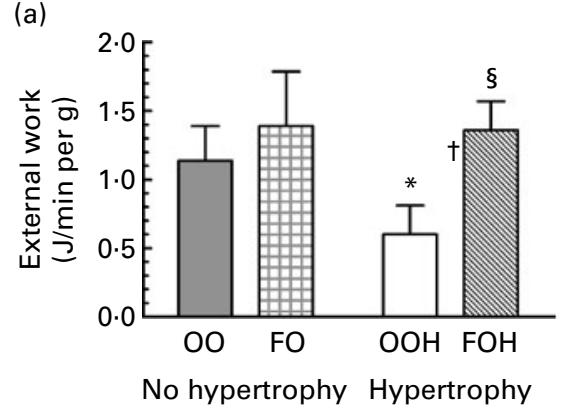

(b)

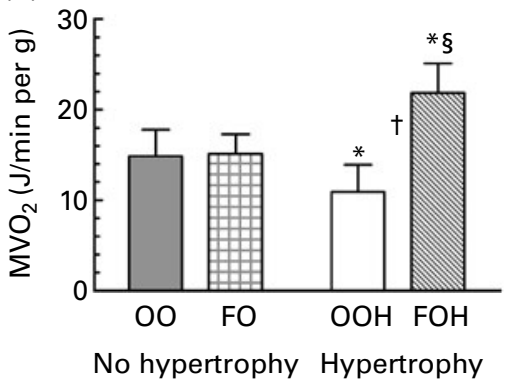

(c)

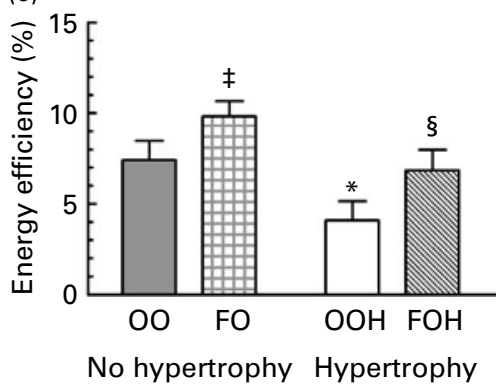

Fig. 1. Effects of fish oil (FO) on (a) cardiac external work, (b) myocardial oxygen consumption $\left(\mathrm{MVO}_{2}\right)$ and (c) cardiac energy efficiency in the isolated working heart without hypertrophy (olive oil $(\mathrm{OO} ; \square), \mathrm{FO}(\mathrm{⿴囗十)})$ ) or with hypertrophy $(\mathrm{OOH}(\square), \mathrm{FOH}(\mathbb{\otimes})$ ). Values are means, with their standard errors represented by vertical bars ( $n$ 6-8 per group). * Mean values were significantly different from those of the no-hypertrophy group $(P<0.05$, ANOVA). $†$ Mean values were significantly different for diet $\times$ hypertrophy interaction $(P<0.05$; ANOVA). $¥$ Mean values were significantly different for FO from those of the 00 control diet $(P<0.05$; ANOVA). § Mean values were significantly different for $\mathrm{FOH}$ from those of the $\mathrm{OOH}$ group $(P<0.05$; ANOVA). 
(a)
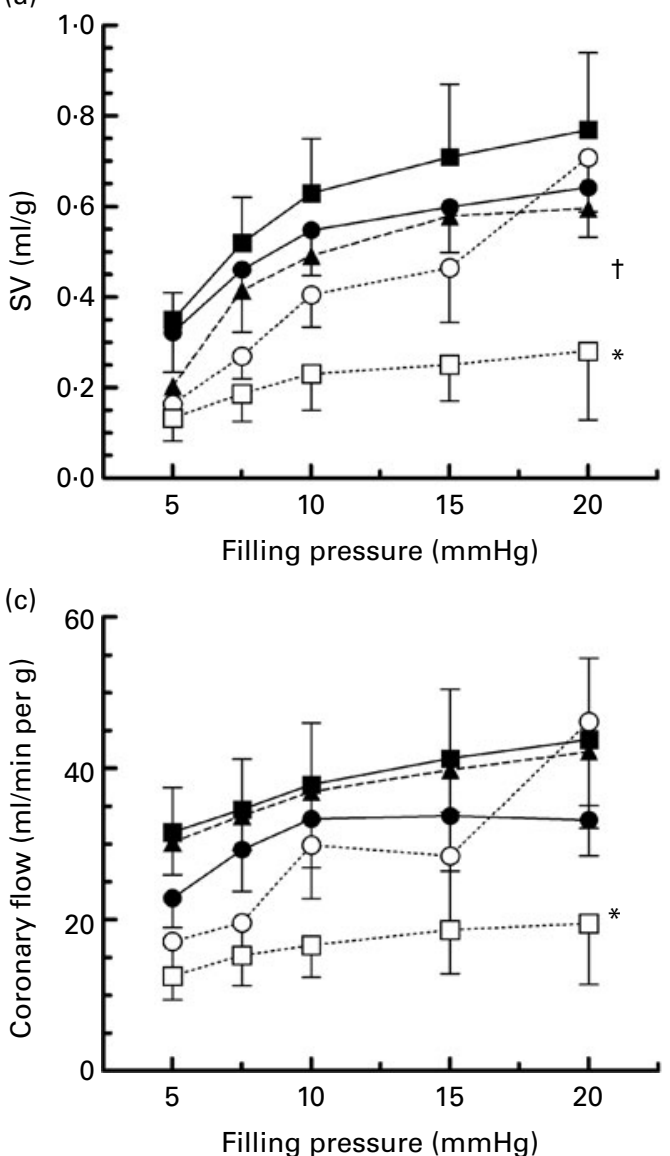

(b)

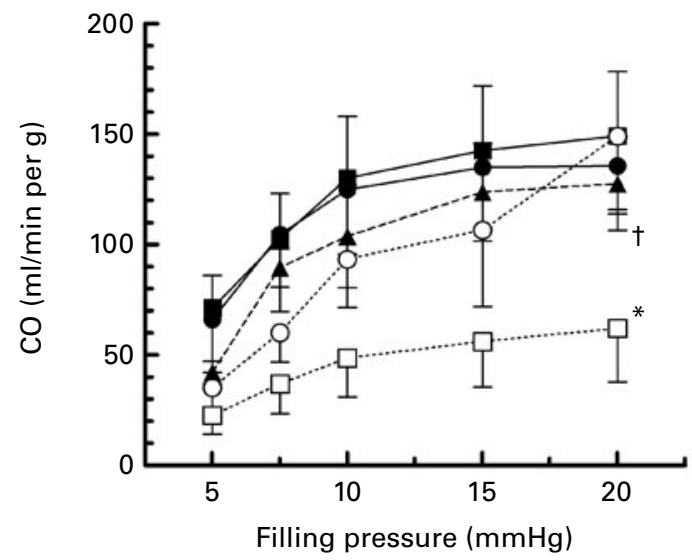

(d)

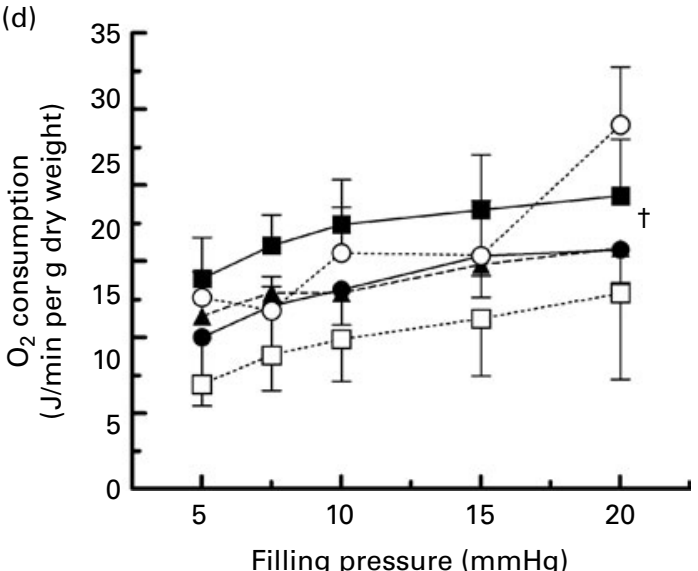

Fig. 2. Effects of fish oil (FO) and cardiac hypertrophy on (a) stroke volume (SV), (b) cardiac output (CO), (c) coronary flow and (d) myocardial oxygen consumption $\left(\mathrm{MVO}_{2}\right)$ in the isolated working heart during changes in ventricular filling pressure: sham-operated olive oil (OO) control diet ( $\boldsymbol{\Delta}$, ---), OO control diet $(\boldsymbol{\square}$, no hypertrophy; $\square$, hypertrophy $(\mathrm{OOH})$ ), FO diet (e, no hypertrophy; $O$, hypertrophy $(\mathrm{FOH})$ ). Values are means, with their standard errors represented by vertical bars ( $n$ 6-8 per group). * Mean values were significantly different from those of the sham OO control $(P<0.05$; ANOVA). $\dagger$ Mean values were significantly different for diet $\times$ hypertrophy interaction $(P<0.05$; ANOVA).

diet significantly increased the concentration of $n-3$ PUFA EPA and DHA and reduced the concentration of monounsaturated oleic acid and the n-6 PUFA arachidonic acid compared with the OO diet (Table 2). The total saturated fat concentration was unaltered but the total PUFA concentration and the unsaturation index were increased with FO feeding.

The hypertrophied $\mathrm{OOH}$ hearts incorporated significantly less linoleic acid $(P<0.05)$ with non-significant trends towards increased arachidonic acid $(P<0 \cdot 1)$ and DHA $(P<0 \cdot 1)$, producing, overall, a non-significant trend towards reduced $n-6$ PUFA $(P<0 \cdot 1)$ and a small but significant increase in unsaturation index $(P<0.05)$ compared with the non-hypertrophied OO hearts (Table 2). There were no significant differences in phospholipid fatty acid composition between the hypertrophied (FOH) and non-hypertrophied (FO) fish oil hearts.

\section{Discussion}

The results of the present study demonstrate that incorporation of $n-3$ PUFA into myocardial cellular membranes to reduce $\mathrm{MVO}_{2}$ in relation to the cardiac work output seen in normal hearts ${ }^{(13)}$ translates into attenuation of cardiac dysfunction in the hypertrophied hearts. Fish oil feeding is known to limit the development of cardiac hypertrophy induced by a variety of stimuli ${ }^{(21,22,24,25)}$, and until now, any attenuation of hypertrophied heart dysfunction by $n$-3 PUFA was always accompanied by, and could not be differentiated from, the prevention of ventricular remodelling ${ }^{(21,24)}$. Our demonstration that hypertrophied heart function was improved independently of attenuating cardiac hypertrophy suggests that dietary fish oil may prevent cardiac dysfunction progressing to heart failure. The improved cardiac function, demonstrated not only at rest (as previously shown in association with hypertrophy attenuation ${ }^{(21,24)}$ ), but over a wide range of loading conditions and functional measures, provides further support.

Fish oil improvement of cardiac function was largely limited to the hypertrophied hearts, even though myocardial energy efficiency was significantly greater with fish oil feeding in the non-hypertrophied hearts. Improved oxygen efficiency often does not translate into cardiac function improvement in healthy, well-oxygenated hearts ${ }^{(13,24)}$. However, 
(a)

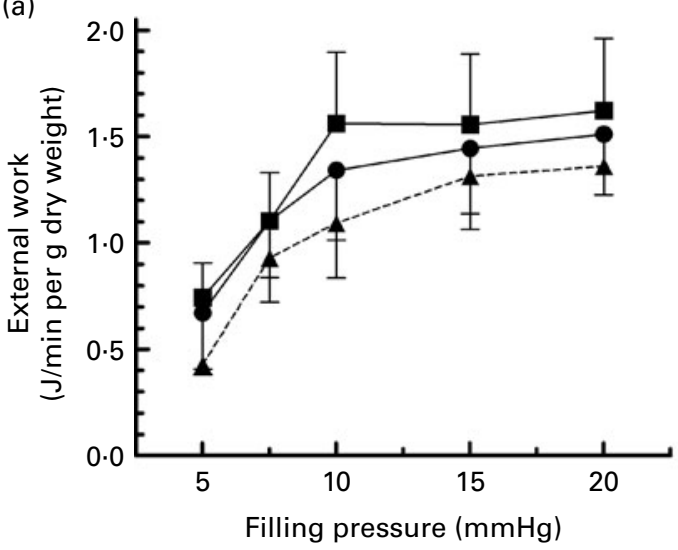

(c)

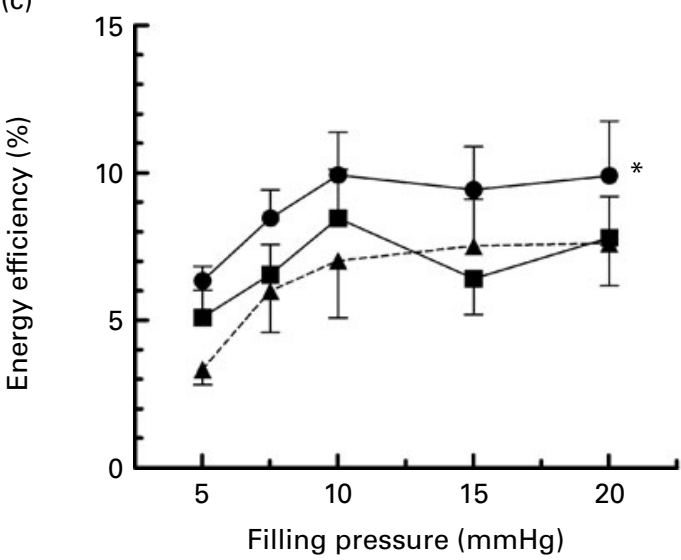

(b)

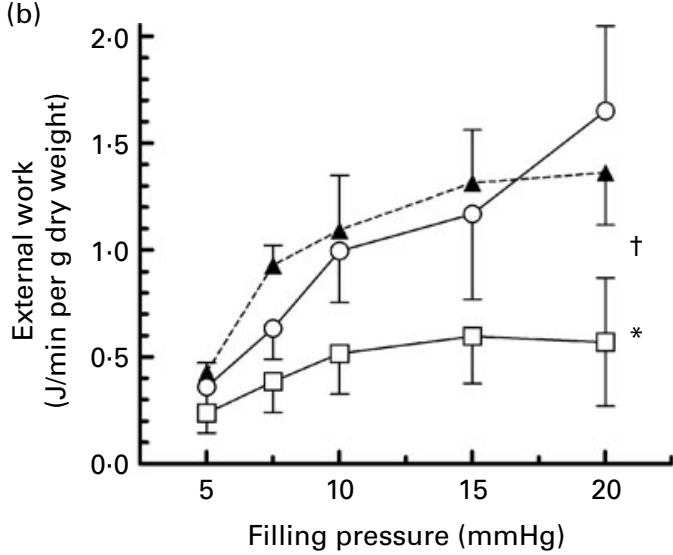

(d)

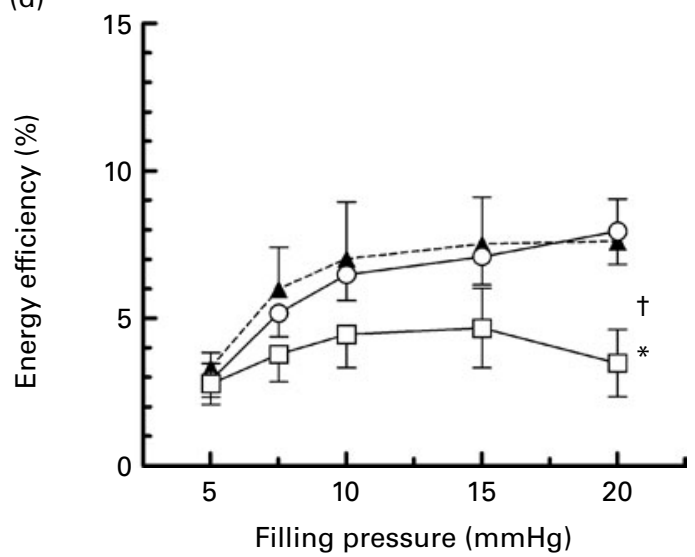

Fig. 3. Effects of dietary oil and cardiac hypertrophy on $(a, b)$ cardiac external work and (c, d) cardiac energy efficiency in the isolated working heart during changes in ventricular filling pressure. (a, c) Sham and non-hypertrophied hearts. (b, d) Sham and hypertrophied hearts. Sham-operated olive oil (OO) control diet $(\boldsymbol{\Lambda},---)$, OO control diet $(\mathbf{\square}$, no hypertrophy; $\square$, hypertrophy $(\mathrm{OOH}))$ and FO diet $(\bullet$, no hypertrophy; O, hypertrophy (FOH)). Values are means, with their standard errors represented by vertical bars ( $n$ 6-8 per group). ${ }^{*}$ Mean values were significantly different from those of the sham 00 control $(P<0.05 ;$ ANOVA). $\dagger$ Mean values were significantly different for diet $\times$ hypertrophy interaction $(P<0.05$; ANOVA).

energy-sparing effects consistently translate into improved heart function under cardio-depressant stress conditions such as myocardial ischaemia ${ }^{(13,16)}$ or cardiac hypertrophy. Attenuation of the decline in $\mathrm{CO}$, the decline in cardiac relaxation and filling, the depressed external work and energy efficiency, over the entire Frank-Starling relationship, especially at high workload, illustrates the potential for translation across all stages of heart failure.

Increased ventricular stiffness impairs relaxation and filling, increases the work of contraction, impairs cardiac energetics, prolongs coronary artery compression and impairs coronary perfusion $^{(18,19,26)}$. It is therefore a major contributor to cardiac dysfunction. Retention of residual wall tension was less evident in the hypertrophied fish oil hearts by virtue of better cardiac relaxation. The hypertrophy-induced reduction in cardiac energy efficiency was attenuated by fish oil feeding, and the hypertrophied fish oil hearts did not display the depressed rate of relaxation, ventricle filling and CO usually associated with post-adaptive cardiac hypertrophy. Furthermore, the increase in coronary vasodilator reserve associated with fish oil in healthy hearts ${ }^{(13,16)}$ translated into an adaptive increase in oxygen delivery in the hypertrophied hearts during the demand of raised filling pressure, overcoming the normally depressed coronary flow and providing further advantage in pathophysiological conditions.

\section{Potential mechanisms of n-3 PUFA actions}

Impaired relaxation, contraction and energetic failure underpinning the pathophysiology of cardiac dysfunction and heart failure are all associated with altered myocardial $\mathrm{Ca}^{2+}$ homeostasis. In turn, energetic failure leads to altered $\mathrm{Ca}^{2+}$ handling, creating a vicious cycle ${ }^{(18)}$. Conversely, numerous effects of the long-chain $n$-3 PUFA on myocardial $\mathrm{Ca}^{2+}$ regulation have biologically plausible correlates in the effects of dietary fish oil on hypertrophied heart function that might play a role in breaking that cycle ${ }^{(14)}$. For example, pathological depolarisation and cardiac arrhythmias may be attenuated by the following: inhibition of phospholipase-C and to thereby attenuate inositol trisphosphate and diacylglycerol mobilisation from membrane phospholipids, which in turn attenuates $\mathrm{Ca}^{2+}$ mobilisation, particularly in response to ischaemic stress; reducing sarcoplasmic reticulum (SR)- $\mathrm{Ca}^{2+}$ overload and attenuating $\mathrm{Ca}^{2+}$ sparks ${ }^{(14,27)}$. Hypertrophied 
With more membrane manufactured as cells enlarge, hypertrophy-driven changes have the potential to alter myocardial phospholipid composition and modify responses to dietary intervention. Indeed, adaptive decreases in linoleic acid and increases in arachidonic acid, DHA and membrane total unsaturation that occur in response to a variety of stressors $^{(38)}$, including pressure overload ${ }^{(20,24)}$ and catecholamine stress $^{(39)}$, were observed in the present study. However, none of the adaptive changes were as great as changes induced by the FO diet. Any hypertrophy-associated increases in DHA and total unsaturation were overwhelmed by the dietary intervention and the arachidonic acid increase was reversed, such that no differences were evident between the hypertrophied and non-hypertrophied fish oil hearts.

\section{Comparison with therapeutic interventions to enhance cardiac function}

Dietary fish oil-induced improvement of hypertrophied heart contractile function has some similarities but many differences to therapeutic interventions used in the treatment of heart failure. For example, positive inotropic agents typically improve systemic haemodynamics and provide symptomatic relief in heart failure by increasing contractility at the expense of reduced energy efficiency ${ }^{(40)}$, increased risk of ischaemia $^{(41)}$, cardiac arrhythmias and increased early mortality. Vasodilators relieve heart failure by reducing systemic blood pressure and unloading the heart, which the fish oil did not do in the present study. Moreover, the negative inotropic actions of the $\mathrm{Ca}^{2+}$ channel blocking class of vasodilators ${ }^{(42)}$ are inconsistent with the effects of fish oil. The angiotensinconverting enzyme (ACE) inhibitors and angiotensin receptor antagonists have no direct effect on myocardial contractile performance, acting principally by inhibiting hypertrophy and myocardial fibrosis ${ }^{(43,44)}$, whereas fish oil directly improved cardiac function without the effect on ventricular remodelling.

The greatest similarity of physiological mechanisms lies with adrenergic $\beta$-blockers, which reduce the heart rate and reduce the chronic hyper-adrenergic state that is typical of heart failure ${ }^{(45)}$, thereby reducing energy requirements. However, although fish oils can reduce intrinsic heart rate and prevent sudden death in rats associated with chronic isoprenaline treatment ${ }^{(39)}$, they have no $\beta$-adrenoceptor antagonist action $^{(46)}$, and unlike $\beta$-blockers, heart rate reduction is achieved independently of sympathetic nervous activity ${ }^{(12,16)}$. In common with $n-3$ PUFA, $\beta$-blockers facilitate chronic intrinsic improvements in cellular function in hypertrophied cardiomyocytes by improvements in cellular energetics ${ }^{(19)}$ or intracellular $\mathrm{Ca}^{2+}$ handling ${ }^{(43)}$.

Thus, dietary fish oil improved cardiac function in hypertrophied hearts in a manner that contrasts to the currently available suite of treatments used to stimulate the heart or counteract dysfunction. It appears to have an intrinsic effect on myocardial cellular function independent of cardiac unloading or slowed heart rate, to improve contractility without adverse energetic consequences and independently of modulating the hypertrophy.

\section{Therapeutic implications}

Epidemiological evidence showing a reduced risk of incident heart failure in people who regularly eat fish ${ }^{(7,47)}$ is now supported by a large secondary prevention clinical trial in which n-3 PUFA supplementation reduced mortality and hospitalisation in heart failure in the context of usual care ${ }^{(48)}$, which included simultaneous treatment with many of the therapeutic agents discussed above. The findings of the present study begin to explain not only the original GISSI-HF outcomes but also a recent subset evaluation of the GISSI-HF trial ${ }^{(48)}$ which noted that the reduced number of hospital admissions due to cardiovascular reasons in heart failure was paralleled by improved cardiac function without the evidence of reverse remodelling ${ }^{(49)}$.

Animal and human studies have suggested that a ceiling exists for the cardiac effects of $n-3$ PUFA $^{(50,51)}$, which in animals reflects maximum incorporation of DHA into myocardial membranes ${ }^{(23,52)}$. Thus, the effects reported here for $5 \%$ dietary fish oil are probably achievable at lower intakes $^{(24,50,53)}$, in line with even very low increases in fish oil intake, commensurate with human dietary intake, to increase myocardial membrane $n-3$ PUFA incorporation ${ }^{(23)}$. An absence of $n$ - 3 PUFA from the diet amplifies the cardiac depressant effects of ischaemic or hypertrophic stress. This can be overcome by incorporating fish oil in the diet to improve myocardial energetics, which is identified as a new candidate approach to the treatment of heart failure ${ }^{(19)}$. When combined with the amelioration of risk for sudden heart attack death and antihypertrophic actions, amelioration of heart failure adds a new dimension to the nutritional preconditioning properties of fish oil fatty acids ${ }^{(16)}$

\section{Acknowledgements}

This study was supported by Roche Vitamins, Basel, Switzerland and by a grant from the National Health and Medical Research Council, Australia (to P. L. M.). Conflict of interest: P. L. M. and M. Y. A. are supported by a research grant from Roche Vitamins, Basel, Switzerland; D. R. is an employee of DSM Nutritional Products, Basel, Switzerland, producer of ROPUFA ${ }^{\circledR} 30 n-3$ EPA oil; J. A. D. has no conflict of interest. All authors contributed to the work. P. L. M. and M. Y. A. conceptualised the study. P. L. M., M. Y. A. and D. R. designed the dietary intervention and P. L. M. designed the experimental model. J. A. D. and P. L. M. conducted the experiments and analysed the data. P. L. M. conducted the statistical analysis. P. L. M. and M. Y. A. interpreted the data. P. L. M. drafted the manuscript. The manuscript was reviewed and approved by all authors.

\section{References}

1. Najafi F, Jamrozik K \& Dobson AJ (2009) Understanding the 'epidemic of heart failure': a systematic review of trends in determinants of heart failure. Eur J Heart Fail 11, 472-479.

2. Bucher HC, Hengstler P, Schindler C, et al. (2002) n-3 Polyunsaturated fatty acids in coronary heart disease: 
a meta-analysis of randomized controlled trials. Am J Med 112, 298-304.

3. Mozaffarian D, Ascherio A, Hu FB, et al. (2005) Interplay between different polyunsaturated fatty acids and risk of coronary heart disease in men. Circulation 111, 157-164.

4. McLennan PL, Abeywardena MY \& Charnock JS (1988) Dietary fish oil prevents ventricular fibrillation following coronary artery occlusion and reperfusion. Am Heart $J$ 116, 709-717.

5. Siscovick DS, Raghunathan TE, King I, et al. (1995) Dietary intake and cell membrane levels of long-chain $n-3$ polyunsaturated fatty acids and the risk of primary cardiac arrest. JAMA 274, 1363-1367.

6. Marchioli R, Barzi F, Bomba E, et al. (2002) Early protection against sudden death by $n-3$ polyunsaturated fatty acids after myocardial infarction: time-course analysis of the results of the GISSI-Prevenzione. Circulation 105, 1897-1903.

7. Mozaffarian D, Bryson CL, Lemaitre RN, et al. (2005) Fish intake and risk of incident heart failure. $\mathrm{J} \mathrm{Am} \mathrm{Coll} \mathrm{Cardiol}$ 45, 2015-2021.

8. Valagussa F, Franzosi MG, Geraci E, et al. (1999) Dietary supplementation with $n-3$ polyunsaturated fatty acids and vitamin $\mathrm{E}$ after myocardial infarction: results of the GISSIPrevenzione trial. Lancet 354, 447-455.

9. Nordoy A, Marchioli R, Arnesen H, et al. (2001) n-3 polyunsaturated fatty acids and cardiovascular diseases. Lipids 36, S127-S129.

10. Burr ML, Fehily AM, Gilbert JF, et al. (1989) Effects of changes in fat, fish, and fibre intakes on death and myocardial reinfarction: diet and reinfarction trial (DART). Lancet $\mathbf{i i}, 757-761$.

11. al Makdessi S, Brandle M, Ehrt M, et al. (1995) Myocardial protection by ischemic preconditioning: the influence of the composition of myocardial phospholipids. Mol Cell Biochem 145, 69-73.

12. Pepe S \& McLennan PL (1996) Dietary fish oil confers direct antiarrhythmic properties on the myocardium of rats. $J$ Nutr 126, 34-42.

13. Pepe $S$ \& McLennan PL (2002) Cardiac membrane fatty acid composition modulates myocardial oxygen consumption and post-ischemic recovery of contractile function. Circulation 105, 2303-2308.

14. McLennan PL \& Abeywardena MY (2005) Membrane basis for fish oil effects on the heart: linking natural hibernators to prevention of human sudden cardiac death. J Memb Biol 206, 85-102.

15. McLennan P, Howe P, Abeywardena M, et al. (1996) The cardiovascular protective role of docosahexaenoic acid. Eur J Pharmacol 300, 83-89.

16. Abdukeyum GG, Owen AJ \& McLennan PL (2008) Dietary $(n-3)$ long-chain polyunsaturated fatty acids inhibit ischemia and reperfusion arrhythmias and infarction in rat heart not enhanced by ischemic preconditioning. $J$ Nutr 138, 1902-1909.

17. Demaison L \& Grynberg A (1994) Cellular and mitochondrial energy metabolism in the stunned myocardium. Basic Res Cardiol 89, 293-307.

18. Mettauer B, Zoll J, Garnier A, et al. (2006) Heart failure: a model of cardiac and skeletal muscle energetic failure. Pflug Arch 452, 653-666.

19. Ormerod JOM, Ashrafian H \& Frenneaux MP (2008) Impaired energetics in heart failure - a new therapeutic target. Pharmacol Ther 119, 264-274.

20. Reibel DK, O'Rourke B, Foster KA, et al. (1986) Altered phospholipid-metabolism in the pressure-overload hypertrophied hearts. Am J Physiol 250, H1-H6.
21. Duda MK, O'Shea KM, Lei B, et al. (2007) Dietary supplementation with omega-3 PUFA increases adiponectin and attenuates ventricular remodeling and dysfunction with pressure overload. Cardiovasc Res 76, 303-310.

22. Siddiqui RA, Shaikh SR, Kovacs R, et al. (2004) Inhibition of phenylephrine-induced cardiac hypertrophy by docosahexaenoic acid. J Cell Biochem 92, 1141-1159.

23. Slee EL, McLennan PL, Owen AJ, et al. (2010) Low dietary fish oil threshold for myocardial membrane n-3 PUFA enrichment independent of $n-6$ PUFA intake in rats. $J$ Lipid Res 51, 1841-1848.

24. Duda MK, O'Shea KM, Tintinu A, et al. (2009) Fish oil, but not flaxseed oil, decreases inflammation and prevents pressure overload-induced cardiac dysfunction. Cardiovasc Res 81, 319-327.

25. Takahashi R, Okumura K, Asai T, et al. (2005) Dietary fish oil attenuates cardiac hypertrophy in lipotoxic cardiomyopathy due to systemic carnitine deficiency. Cardiovasc Res $\mathbf{6 8}$, 213-223.

26. Traverse JH, Chen Y, Crampton M, et al. (2001) Increased extravascular forces limit endothelium-dependent and -independent coronary vasodilation in congestive heart failure. Cardiovasc Res 52, 454-461.

27. Siddiqui RA, Harvey KA \& Zaloga GP (2008) Modulation of enzymatic activities by $n-3$ polyunsaturated fatty acids to support cardiovascular health. J Nutr Biochem 19 417-437.

28. Pepe S, Tsuchiya N, Lakatta EG, et al. (1999) PUFA and aging modulate cardiac mitochondrial membrane lipid composition and $\mathrm{Ca}^{2+}$ activation of PDH. Am J Physiol 276, H149-H158.

29. Hallaq H, Sellmayer A, Smith TW, et al. (1990) Protective effect of eicosapentaenoic acid on ouabain toxicity in neonatal rat cardiac myocytes. Proc Natl Acad Sci 87, $7834-7838$.

30. Leifert WR, Dorian CL, Jahangiri A, et al. (2001) Dietary fish oil prevents asynchronous contractility and alters $\mathrm{Ca}^{2+}$ handling in adult rat cardiomyocytes. $J$ Nutr Biochem 12, 365-376.

31. McLennan PL (2001) Myocardial membrane fatty acids and the antiarrhythmic actions of dietary fish oil in animal models. Lipids 36, S111-S114.

32. Sexton PT, Sinclair AJ, O'Dea K, et al. (1995) The relationship between linoleic acid level in serum, adipose tissue and myocardium in humans. Asia Pacific J Clin Nutr $\mathbf{4}$, 314-318.

33. Calder PC (2007) Immunomodulation by omega-3 fatty acids. Prostaglandins Leukot Essent Fatty Acids 77, 327-335.

34. Mozaffarian D, Geelen A, Brouwer IA, et al. (2005) Effect of fish oil on heart rate in humans - a metaanalysis of randomized controlled trials. Circulation 112, 1945-1952.

35. Yokoyama M, Origasa H, Matsuzaki M, et al. (2007) Effects of eicosapentaenoic acid on major coronary events in hypercholesterolaemic patients (JELIS): a randomised openlabel, blinded endpoint analysis. Lancet 369 1090-1098.

36. Abeywardena MY \& Charnock JS (1995) Dietary lipid modification of myocardial eicosanoids following ischemia and reperfusion in the rat. Lipids 30, 1151-1156.

37. Abeywardena MY, McLennan PL \& Charnock JS (1991) Changes in myocardial eicosanoid production following long-term dietary lipid supplementation in rats. Am J Clin Nutr 53, 1039S-1041S.

38. Novak F, Kolar F, Hamplova B, et al. (2009) Myocardial phospholipid remodeling under different types of load 
imposed during early postnatal development. Physiol Res 58, $\mathrm{S} 13-\mathrm{S} 32$

39. Gudbjarnason S, Benediktsdottir VE \& Skuladottir G (1989) Effects of $n-3$ polyunsaturated fatty acids on coronary heart disease. Bibliotheca Nutritio et Dieta 43, 1-12.

40. Nikolaidis LA, Trumble D, Hentosz T, et al. (2004) Catecholamines restore myocardial contractility in dilated cardiomyopathy at the expense of increased coronary blood flow and myocardial oxygen consumption $\left(\mathrm{MVO}_{2}\right.$ cost of catecholamines in heart failure). Eur J Heart Fail 6, 409-419.

41. Katz AM (1986) Potential deleterious effects of inotropic agents in the therapy of chronic heart-failure. Circulation 73, 184-190.

42. Lubic SP, Giacomini KM \& Giacomini JC (1995) The effects of modulation of calcium influx through the voltage-sensitive calcium-channel on cardiomyocyte hypertrophy. J Mol Cell Cardiol 27, 917-925.

43. Eichhorn EJ \& Bristow MR (1996) Medical therapy can improve the biological properties of the chronically failing heart. A new era in the treatment of heart failure. Circulation 94, 2285-2296.

44. Kim S, Yoshiyama M, Izumi Y, et al. (2001) Effects of combination of ACE inhibitor and angiotensin receptor blocker on cardiac remodeling, cardiac function, and survival in rat heart failure. Circulation 103, 148-154.

45. Nagatsu M, Spinale FG, Koide M, et al. (2000) Bradycardia and the role of $\beta$-blockade in the amelioration of left ventricular dysfunction. Circulation 101, 653-659.
46. Reibel DK, Holahan MA \& Hock CE (1988) Effects of dietary fish oil on cardiac responsiveness to adrenoceptor stimulation. Am J Physiol 254, H494-H499.

47. Levitan EB, Wolk A \& Mittleman MA (2009) Fish consumption, marine omega-3 fatty acids, and incidence of heart failure: a population-based prospective study of middleaged and elderly men. Eur Heart J 30, 1495-1500.

48. Tavazzi L, Maggioni AP, Marchioli R, et al. (2008) Effect of $n-3$ polyunsaturated fatty acids in patients with chronic heart failure (the GISSI-HF trial): a randomised, double-blind, placebo-controlled trial. Lancet 372, 1223-1230.

49. Ghio S, Scelsi L, Latini R, et al. (2010) Effects of $n$-3 polyunsaturated fatty acids and of rosuvastatin on left ventricular function in chronic heart failure: a substudy of GISSI-HF trial. Eur J Heart Fail 12, 1345-1353.

50. McLennan PL, Owen AJ, Slee EL, et al. (2007) Myocardial function, ischaemia and $n-3$ polyunsaturated fatty acids: a membrane basis. J Cardiovasc Med 8, Suppl. 1, S15-S18.

51. Mozaffarian D \& Rimm EB (2006) Fish intake, contaminants, and human health - evaluating the risks and the benefits. JAMA 296, 1885-1899.

52. Owen AJ, Peter-Przyborowska BA, Hoy AJ, et al. (2004) Dietary fish oil dose- and time-response effects on cardiac phospholipid fatty acid composition. Lipids 39, 955-961.

53. Pepe S \& McLennan P (2007) (n-3) Long chain PUFA dose-dependently increase oxygen utilization efficiency and inhibit arrhythmias after saturated fat feeding in rats. J Nutr 137, 2377-2383. 